\title{
Can Irrational Numbers (Such as Square Root of the Number Five) Be Reached by Analysis of Genetic Sequences?
}

\author{
Tahir Ölmez \\ Social Sciences Dept., Selçuk University, Konya, Turkey \\ Email: bsonmez3@gmail.com, tolmez123@yahoo.com
}

How to cite this paper: Ölmez, T. (2021) Can Irrational Numbers (Such as Square Root of the Number Five) Be Reached by Analysis of Genetic Sequences? Open Access Library Journal, 8: e7104.

https://doi.org/10.4236/oalib.1107104

Received: December 22, 2020

Accepted: January 23, 2021

Published: January 26, 2021

Copyright $\odot 2021$ by author(s) and Open Access Library Inc.

This work is licensed under the Creative Commons Attribution International License (CC BY 4.0).

http://creativecommons.org/licenses/by/4.0/

\section{(c) (i) Open Access}

\begin{abstract}
Irrational numbers didn't have reoccurrence sequence but this paper calculated a sequence with respect to Quantum Perspective Model. One of the irrational numbers is the square root of five numbers. This article researches whether there is a link between the square root of five numbers and the genetic sequences. At first, the square root digits of the number five after the comma are added respectively. Secondly, the resulting sum corresponds to the nucleotide bases, the results obtained in this way are expressed as nucleotide bases (A, T, C, G, and U): (A) Adenine, (T) Thymine, (C) Cytosine, (G) Guanine, (U) Uracil. From this point of view, when the first three hundred digits of the square root of the number five after the comma are calculated, the gene sequence is obtained as follows: [ATTTATTCAATACATAACCCCATTGA]. Thirdly, in this sequence, some of reoccurrences were detected just like as "CAT" and "ATT". Fourthly, after researching this sequence at NCBI (National Biotechnology Information Center), the search result is similar to bony fishes, especially DANIO RERIO (Zebra fish). Lastly, the genetic codes of Zebra fishes were found to be similar to human genetic codes. In summary, the connection between these results and the square root of the five in mathematical science and the genetic codes in biochemistry may shed light on explaining irrational numbers.
\end{abstract}

\section{Subject Areas}

Mathematical Logic and Foundation of Mathematics

\section{Keywords}

Quantum Perspective Model, Biochemistry, DANIO RERIO (Zebra Fish), The Square Root of Five and NCBI (National Biotechnology Information Center) 


\section{Introduction}

Irrational numbers have no decimal expansions that end and become periodic [1]. But this paper calculated many reoccurences in the calculation of the square root of five numbers [2] just like as "CAT" [(C) Cytosine, (A) Adenine and (T) Thymine] and "ATT" [(A) Adenine, (T) Thymine and (T) Thymine] [ATTTATTCAATACATAACCCCATTGA]. Before this study, some of the irrational numbers were researched as in pi numbers [3], the golden ratio numbers [4], Euler's numbers [5], the square root of number two [6] and the square root of number three [7]. According to Quantum Perspective Model, the meaning of Pi number is forever UTA's [(U) Uracil, (T) Thymine and (A) Adenine]. Also the sum of chemical formulas of TATA and CAAT Box is near as the same as the golden mean numbers of "618" (Remember, approximately the golden ratio is 1,618). Besides, after the comma, Euler's eighteen fifteen-group numbers are converted to nucleotide bases. The results obtained in this way are expressed as nucleotide bases as follows: "AUGUUGAUAUTAAUCATC". Nextly, the calculation of the square root of number two is "GGATGTCTATTGAGTGACAA". Lastly, the calculation of the square root of number three is "GGATGACTACGGGTTTAGAAA". In sum, all of these explained irrational numbers have a genetic sequence. Let me see these similarities of irrational numbers with respect to genetic sequences.

\section{Methods and Discussion}

In this work, the chemical formulas of nucleotide bases are calculated with regards to atomic numbers of elements. The chemical structures of bases include Carbon (C), Nitrogen (N), Oxygen (O), and Hydrogen (H). Calculation of bases with chemical atoms (See also Table 1) (Ölmez T, 2020) [4].

In this research, genetic codes were used as a new formula (Nirenberg et al., 1965) [10]. just like as (A, T, C, G, and U). (A) Adenine, (T) Thymine, (C) Cytosine, (G) Guanine, (U) Uracil. Prior to this study, a paper containing $c$ is regulatory elements (TATA box, CAAT box and GC box) shed lights on the links between the Golden Ratio numbers and chemical formulas [4]. Then, according to the Quantum Perspective Model, the connection between the square root of the two numbers and the genetic codes was studied [6]. Nextly, the relationships between the square root of number three [7] and genetic codes were researched. Lastly, The connection between Euler numbers and the Fibonacci series was examined [11]. Now, the square root of the number five and its genetic codes are calculated.

\section{Calculation of the Square Root of Five Numbers and Genetic Codes}

The first three hundred digits of the square root of five after the comma are here: the square root of $5=$

2.2360679774997896964091736687312762354406183596115257242708972454 
10520925637804899414414408378782274969508176150773783504253267724447 07386358636012153345270886677817319187916581127664532263985658053576 13504175337850034233924140644420864325390972525926272288762995174024 40681611775908909498492371390729728898482088641542689894099131693577 0197486788844250897541329561831769 [2].

At first, the first group of the square root numbers of five after comma was taken. For example, 2, 3, 6, 0, 6, 7, 9, 7, 7, 4, 9, $9 \ldots$ and so on. Secondly, all decimal numbers are subjected to the addition process, respectively. $(2+3+6+$ $0+6+7+9+7+7+4+9+9=69)$. The sum of the first group of the root square numbers of five after comma is "69". Just like as in (A) Adenine: 70 (See also Table 1).

The first group of the root square numbers of five after comma: $2+3+6+0+6+7+9+7+7+4+9+9=69$ (A) Adenine: 70

The second group of the root square numbers of five after comma: $7+8+9+6+9+6+4+0+9+1+7=66(\mathrm{~T})$ Thymine: 66

The third group of the root square numbers of five after comma: $3+6+6+8+7+3+1+2+7+6+2+3+5+4+4+0=67(\mathrm{~T})$ Thymine: 66 The fourth group of the root square numbers of five after comma: $6+1+8+3+5+9+6+1+1+5+2+5+7+2+4+2=67$ (T) Thymine: 66 The fifth group of the root square numbers of five after comma:

$7+0+8+9+7+2+4+5+4+1+0+5+2+0+9+2+5=70$ (A) Adenine: 70

The sixth group of the root square numbers of five after comma: $6+3+7+8+0+4+8+9+9+4+1+4+4=67$ (T) Thymine: 66 The seventh group of the root square numbers of five after comma: $1+4+4+0+8+3+7+8+7+8+2+2+7+4=65$ (T) Thymine: 66 The eighth group of the root square numbers of five after comma: $9+6+9+5+0+8+1+7+6+1+5+0+7=64(\mathrm{C})$ Cytosine: 64 The tenth group of the square numbers of five after comma: $7+3+7+8+3+5+0+4+2+5+3+2+6+7+7=69$ (A) Adenine: 70 The eleventh group of the root square numbers of five after comma: $2+4+4+4+7+0+7+3+8+6+3+5+8+6+3=70$ (A) Adenine: 70

Table 1. Representation of nucleotide bases (A, T, C, G and U) in chemical atoms.

\begin{tabular}{cccccc}
\hline ATOMS /NUCLEOTIDE BASES & $C=6$ & $H=1$ & $\boldsymbol{O}=\boldsymbol{8}$ & $\boldsymbol{N}=7$ & SUM \\
\hline ADENINE: C5H5N5 & 5 & 5 & - & 5 & 70 \\
THYMINE: C5H6N2O2 & 5 & 6 & 2 & 2 & 66 \\
CYTOSINE: C4H5N3O1 & 4 & 5 & 1 & 3 & 64 \\
GUANINE: C5H5N5O1 & 5 & 5 & 1 & 5 & 78 \\
URACIL: C4H4N2O2 & 4 & 4 & 2 & 2 & 58 \\
\hline
\end{tabular}

The atomic numbers of them: Carbon (C): 6, Nitrogen (N): 7, Oxygen (O): 8, Hydrogen (H): 1 (Wieser E M et al., 2013) [8]. The chemical structures of bases (A, T, C, G, and U) are shown at below (Ölmez T, 2020) [4]. (A) Adenine: C5H5N5: 70; (T) Thymine: C5H6N2O2: 66, (C) Cytosine: C4H5N3O1: 64, (G) Guanine: C5H5N5O1: 78, and (U) Uracil: C4H4N2O2: 58 (Lodish $\mathrm{H}$ et al., 2018) [9]. 
The twelfth group of the root square numbers of five after comma:

$6+0+1+2+1+5+3+3+4+5+2+7+0+8+8+6+6+7=65$ (C)

Cytosine: 64

The thirteenth group of the root square numbers of five after comma:

$7+8+1+7+3+1+9+1+8+7+9+1=69$ (A) Adenine: 70

The fourteenth group of the root square numbers of five after comma: $6+5+8+1+1+2+7+6+6+4+5+3+2+2+6=64$ (C) Cytosine: 64 The sixteenth group of the root square numbers of five after comma: $3+9+8+5+6+5+8+0+5+3+5+7+6=70$ (A) Adenine: 70 The seventh group of the root square numbers of five after comma: $1+3+5+0+4+1+7+5+3+3+7+8+5+0+0+3+4+2+3=66$

(T) Thymine: 66

The eighteenth group of the root square numbers of five after comma: $3+9+2+4+1+4+0+6+4+4+4+2+0+8+6+4+3+2+5=70$

(A) Adenine: 70

The nineteenth group of the root square numbers of five after comma: $3+9+0+9+7+2+5+2+5+9+2+6+2+7+2=70$ (A) Adenine: 70 The twenth group of the root square numbers of five after comma: $2+8+8+7+6+2+9+9+5+1+7+4+0+2=64$ (C) Cytosine: 64 The twentyoneth group of the root square numbers of five after comma: $4+4+0+6+8+1+6+1+1+7+7+5+9=61$ (C) Cytosine: 64 The twentytwoth group of the root square numbers of five after comma: $0+8+9+0+9+4+9+8+4+9+2+3=65$ (T) Thymine: 66 The twentythreeth group of the root square numbers of five after comma: $7+1+3+9+0+7+2+9+7+2+8+8=63$ (C) Cytosine: 64

The twenty fourth group of the root square numbers of five after comma: $9+8+4+8+2+0+8+8+6+4+1+5+4+2=69$ (A) Adenine: 70 The twenty-fifth group of the root square numbers of five after comma: $6+8+9+8+9+4+0+9+9+1+3=66(\mathrm{~T})$ Thymine: 66

The twenty sixth group of the root square numbers of five after comma: $1+6+9+3+5+7+7+0+1+9+7+4+8=67(\mathrm{~T})$ Thymine: 66 The twenty seventh group of the root square numbers of five after comma: $6+7+8+8+8+4+4+2+5+0+8+9+7=76$ (G) Guanine: 78 The twenty eighth group of the root square numbers of five after comma: $5+4+1+3+2+9+5+6+1+8+3+1+7+6+9=70$ (A) Adenine: 70 This sequence can be shown as [ATTTATTCAATACATAACCCCATTGA]. Let me try to explain this sequence with the "Quantum Perspective Model." For example, The first group of the square root of five after comma equal to Adenine (A): 69 with the lack of one "1" Hydrogen bond (H:1). (Remember, See Table 1; Adenine (A): 70) This result may mean the sequence of the square root of five in groups [ATTTATTCAATACATAACCCCATTGA]. The third group of the square root of five after the comma is regarded as Thymine $(\mathrm{T})$ with one more Hydrogen bond (H:1); Thymine (T): 67. (Remember, See Table 1; Thymine (T): 66) (Because the deviations in the calculation of the square root of 
five numbers can be derived from the Adenine (A) Thymine (T) Hydrogen bonds because of Adenine (A) pairs with Thymine (T) by two hydrogen bonds. Cytosine (C) Guanine (G) pairs with by three hydrogen bonds [3]. The reason for the lack of hydrogen bonds: Hydrogen bonding is a very versatile attraction (Ölmez T, 2020) [4]. Hydrogen bonds are relatively weak and easily broken by increasing hardness (Farrell R E, 2010) [12].

\section{Results}

After searching the square root of the number five with the National Biotechnology Information Center (NCBI) databases, some conceptual relationships with bony fishes can ultimately be found. Types of bony fishes are based on DANIO RERIO (Zebra fish) (See Figure 1). Types of bony fishes are pinecone soldier fish, orbiculate cardinal fish and blunt-snouted clingfish. Types of other living creatures are wild silkworm, cotton bollworm, zig-zag eel, house mouse, pacific white-sided dolphin, common bottlenose dolphin, radish, domestic cat, blue whale, killer whale, beluga whale, long-finned pilot whale, chickpea, cowpea, field mustard, tomato, potato and wine grape [13] (See Figure 2).

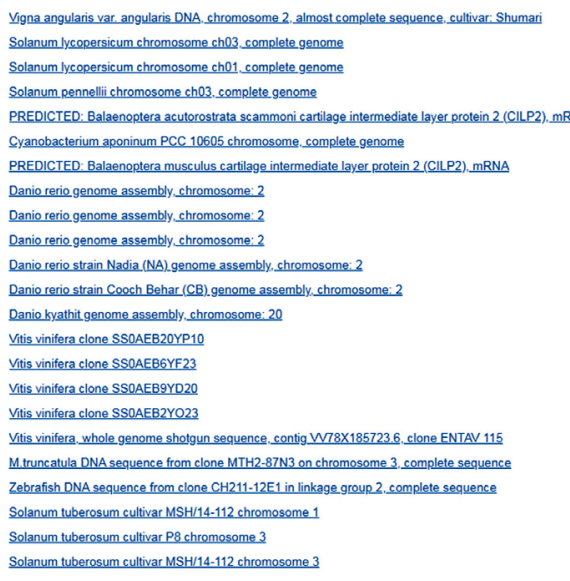

\begin{tabular}{|c|c|c|c|c|c|c|c|}
\hline Vigna angular... & 36.2 & 68.4 & $73 \%$ & 22 & $100.00 \%$ & 45515668 & AP015035.1 \\
\hline tomato & 36.2 & 36.2 & $69 \%$ & 22 & $100.00 \%$ & & 5515.1 \\
\hline omato & 36.2 & 36.2 & $69 \%$ & 22 & $100.00 \%$ & 90311507 & HG975513.1. \\
\hline Solanum pen... & 36.2 & 100 & $73 \%$ & 22 & $100.00 \%$ & 75414019 & HG975442.1. \\
\hline Salaenoptera... & 36.2 & 36.2 & $84 \%$ & 22 & $95.45 \%$ & 3872 & XM_007195407.1 \\
\hline Cyanobacteri... & 362 & 36.2 & $69 \%$ & 22 & $100.00 \%$ & 4114099 & CP003947.1 \\
\hline Blue whale & 36.2 & 36.2 & $84 \%$ & 22 & $95.45 \%$ & 3878 & XM_ 0368453671 \\
\hline zebrafish & 36.2 & 36.2 & $69 \%$ & 22 & $100.00 \%$ & 59970185 & $\lfloor R 812064.1$ \\
\hline zebrafish & 36.2 & 36.2 & $69 \%$ & 22 & $100.00 \%$ & 57662316 & LR812039.1 \\
\hline zebrafshn & 36.2 & 36.2 & $69 \%$ & 22 & $100.00 \%$ & 58067448 & $\lfloor$ LR812595.1 \\
\hline zebrafish & 36.2 & 36.2 & $69 \%$ & 22 & $100.00 \%$ & 59275206 & $\lfloor R 812570.1$ \\
\hline rafish & 36.2 & 36.2 & $69 \%$ & & $100.00 \%$ & 54745004 & $\lfloor$ LR812545.1 \\
\hline Danio kyathit & 36.2 & 68.4 & $76 \%$ & 22 & $100.00 \%$ & 64228523 & $\lfloor$ LR812538.1 \\
\hline wine grape & 36.2 & 36.2 & $69 \%$ & 22 & $100.00 \%$ & 1379 & FO3 090060.1 \\
\hline wine grape & 36.2 & 36.2 & $69 \%$ & 22 & $1000.00 \%$ & 1469 & F0397578.1 \\
\hline wine grape & 36.2 & 36.2 & $69 \%$ & 22 & $100.00 \%$ & 1285 & F0396660.1 \\
\hline negrape & 36.2 & 36.2 & $69 \%$ & 22 & $100.00 \%$ & 1366 & EQ386129.1 \\
\hline wine grape & 36.2 & 36.2 & $69 \%$ & 22 & $100.00 \%$ & 10079 & AM476488.1 \\
\hline barrel medic & 36.2 & 70.4 & $69 \%$ & 22 & $100.00 \%$ & 104115 & Cт961059.9 \\
\hline zebrafish & 36.2 & 36.2 & $69 \%$ & 22 & $100.00 \%$ & 158932 & BX000484.12 \\
\hline tate & 32.2 & 32.2 & $61 \%$ & 347 & $100 . .00 \%$ & 88648484 & СP046702.1 \\
\hline Alu & 32.2 & 32.2 & $69 \%$ & 347 & $94.44 \%$ & 6226726 & 046682.1 \\
\hline olato & 32.2 & 32.2 & $69 \%$ & 347 & $94.44 \%$ & 62282962 & \\
\hline
\end{tabular}

Figure 1. The NCBI (National Biotechnology Information Center) result for nucleotide sequence "ATTTATTCAATACATAACCCCATTGA" [13].

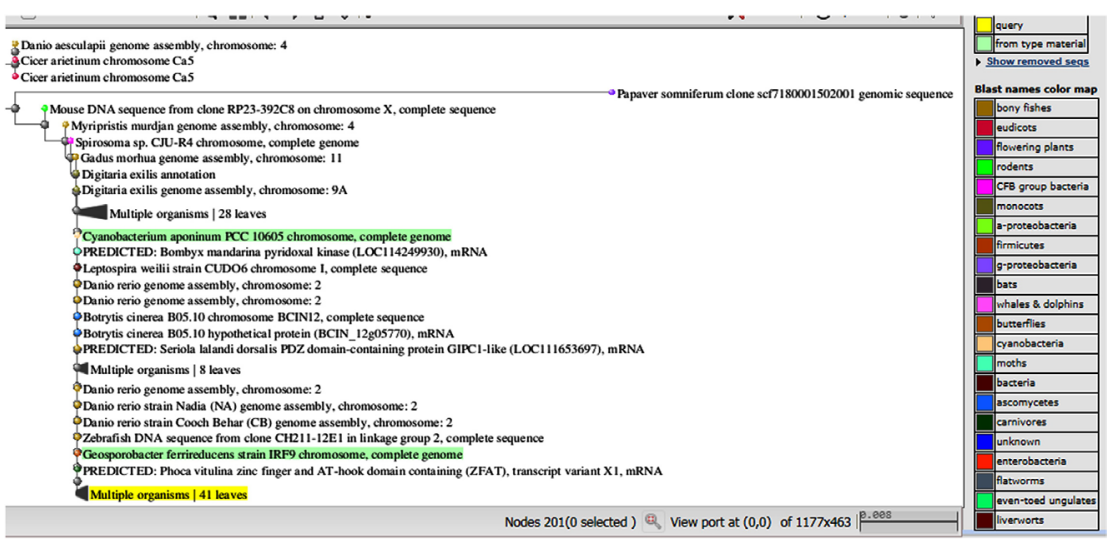

Figure 2. The NCBI (National Biotechnology Information Center) result blast tree view widget for “ATTTATTCAATACATAACCCCATTGA" nucleotide sequence [13]. 


\section{Conclusion}

First, the result of this research can be summarized as the expression of root five numbers after commas by genetic codes. Secondly, digits after the comma were thought to be an indicator of genetic codes. As a common feature of Biochemistry and Mathematical sciences, NCBI blasts are obtained [13]. Because these results include both bony fishes and especially DANIO RERIO (Zebra fish) (See Figure 1). In biology-related experiments and gene sequencing, it is an excellent favorite example [14]. The common feature of pi numbers [3] and Euler numbers [5] along with the square of the speed of light [15] is that the NCBI results are bony fish [13]. Let alone this result, NCBI result for CAAT Box also consists of bony fishes, too (See Figure 2) [4]. Thirdly, with the genetic codes of the square root of two [6] and the genetic codes of the square root of three consist of the same NCBI blast results as the same as bony fishes [7]. Fourthly, although irrational numbers don't have a periodic sequence, this paper finds a way to gain a periodic sequence with regards to genetic sequences just like as in "CAT" and "ATT" (Remember, This sequence can be shown as [ATTTATTCAATACATAACCCCATTGA]. Fifthly, with respect to genetic sequences, not only the calculation of the square root of number two [6] has "GGATG", but also the calculation of the square root of number three [7] has "GGATG", too. These similarities may shed light on explaining irrational numbers in future works. Finally, the results of calculating the square root of five numbers with genetic codes can be expressed by chemical formulas of nucleotide bases. It is stated by Steward that life is between genes and Mathematics (Stewart I, 1999) [16]. Indeed, the relationships between Mathematics and Genetics have been described by a genetic alphabet system. Also, can the square root of the number five be actively used in the analysis of genetic sequences? (Petoukhov S V, 2011) [17].

\section{Conflicts of Interest}

The author declares no conflicts of interest regarding the publication of this paper.

\section{References}

[1] Irrational Number. https://mathworld.wolfram.com/IrrationalNumber.html

[2] https://apod.nasa.gov/htmltest/gifcity/sqrt5.1mil

[3] Köklü, K. (2019) A Quantum Perspective Model to Genetic Codes through Various Sciences. NeuroQuantology, 17, 15-18. https://doi.org/10.14704/nq.2019.17.3.1974

[4] Ölmez, T. (2020) Is There an Aesthetics in Golden Ratio as Regards to the Common Cis-Regulatory Elements versus to Atomic Numbers of Elements with Respect to Quantum Perspective Model? Neurology and Neuroscience Reports, 3, 1-4.

[5] Ölmez, T. (2021) With Respect to Quantum Perspective Model, Can Euler Numbers Be Related to Biochemistry? Global Journal of Science Frontier Research, 20, 7-14. https://doi.org/10.34257/GJSFRFVOL20IS9PG7

[6] Ölmez, T. (2020) According to the Binary Number Base System, Does the Square 
Root of the Number Two Mean in Biochemistry?

[7] Ölmez, T. (2020) What Is the Meaning of the Square Root of the Number Three in Biochemistry?

[8] Wieser, E.M., Holden, N., Coplen, B.T., Böhlke, J.K., Berglund, M., Brand, W.A., et al. (2013) Atomic Weights of the Elements 2011. Pure and Application Chemistry, 85, 1047-1078. https://doi.org/10.1351/PAC-REP-13-03-02

[9] Lodish, H., Berk, A., Zipursky, S.L., Matsudaira, P., Baltimore, D. and Darnell, J. (2018) Molecular Cell Biology. 6th Edition, Translation: Geçkil, H., Özmen, M., Yeşilada, Ö., Palme Publishing, New York, 294-302.

[10] Nirenberg, M., Leder, P., Bernfield, M., Brimacombe, R., Trupin, J., Rottman, F. and O’Neal, C. (1965) RNA Codewords and Protein Synthesis, VII. On the General Nature of the RNA Code. Proceedings of the National Academy of Sciences of the United States of America, 53, 1161-1168. https://doi.org/10.1073/pnas.53.5.1161

[11] Ölmez, T. (2021) Is There a Similarity between Fibonacci Sequence and Euler's Number with Respect to Quantum Perspective Model? Global Journal of Science Frontier Research, 20, 33. https://doi.org/10.34257/GJSFRFVOL20IS9PG35

[12] Farrell, R.E. (2010) RNA Methodologies A Laboratory Guide for Isolation and Characterization. 4th Edition, Elsevier Academic Press, Amsterdam, 704-710.

[13] https://blast.ncbi.nlm.nih.gov/Blast.cgi

[14] https://en.wikipedia.org/wiki/Zebrafish

[15] Köklü, K. (2019) Is Relativity Theory Also Valid in Biogenetics and Mathematics? NeuroQuantology, 17, 53-58. https://doi.org/10.14704/nq.2019.17.3.1999

[16] Stewart, I. (1999) Life's Other Secret: The New Mathematics of the Living World. Penguin, New York.

[17] Petoukhov, S.V. (2011) Matrix Genetics and Algebraic Properties of the Multi-Level System of Genetic Alphabets. NeuroQuantology, 9, 60-81.

https://doi.org/10.14704/nq.2011.9.4.501 